\title{
A New Diagnostic Criterion with Gadoxetic Acid-Enhanced MRI May Improve the Diagnostic Performance for Hepatocellular Carcinoma
}

\author{
Yi Wei $^{a}$ Zheng Yea Yuan Yuan ${ }^{a}$ Zixing Huang ${ }^{a}$ Xiaocheng Wei ${ }^{b}$ \\ Tong Zhang $^{a}$ Shang Wan ${ }^{a}$ Hehan Tang $^{a}$ Xiaopeng He ${ }^{c}$ Bin Song ${ }^{a}$ \\ a Department of Radiology, West China Hospital, Sichuan University, Chengdu, China;

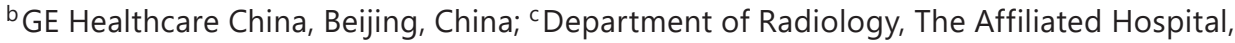 \\ Southwest Medical University, Luzhou, China
}

\section{Keywords}

Diagnostic criterion · Diagnostic performance - Gadoxetic acid enhanced · Hepatocellular carcinoma $\cdot$ Magnetic resonance imaging

\begin{abstract}
Background: To prospectively establish and validate new diagnostic criterion (DC) for liverspecific contrast agents and further compared the diagnostic sensitivity and specificity with conventional DC. Methods: Institutional Review Board approved and written informed consent were obtained for this prospective study. Two board-certified reviewers established the reference standard as hepatocellular carcinoma (HCC), non-HCC lesions by using marks on all cross-sectional MR images. Another 2 abdominal radiologists independently performed the marked lesion observations using 5 different DCs, including DC-1: arterial phase hyperenhancement (APHE) and portal venous phase washout; DC-2: APHE and hepatobiliary phase (HBP) hypointensity; DC-3: APHE and diffusion-weighted imaging (DWI) hyperintensity; DC-4: HBP hypointensity and DWI hyperintensity; DC-5: HBP hypointensity, DWI hyperintensity and excluded these markedly T2 hyperintensity. Diagnostic performance of sensitivity, specificity, and accuracy for each imaging DC was calculated, per-lesion diagnostic sensitivity and specificity of imaging criteria were compared by using McNemars test. Results: A total of 215 patients were included (mean age $53.82 \pm 11.24$ years; range $24-82$ years) with 265 hepatic nodules (175 HCCs and 90 non-HCCs). The DC-4 (93.71\%; 164/175) and DC-5 (92.57\%; 162/175) yielded the highest diagnostic sensitivity and was better than DC-1 $(72.57 \% ; 127 / 175), D C-2$ (82.86\%; 145/175), and DC-3 (82.29\%; 144/175) (all $p<0.001)$. The specificity of DC-1 $(94.44 \%$;
\end{abstract}

Y.W., Z.Y., and Y.Y. contributed equally to this work. 
85/90) was significantly higher than that with DC-2 (83.33\%; 75/90), DC-3 (84.44\%; 76/90), DC-4 (74.44\%; 67/90), and DC-5 (82.22\%; 74/90) (all $p<0.05)$. Additionally, the DC-4 and DC-5 achieved the highest area under curve value of $0.841(95 \% \mathrm{Cl} 0.783-0.899)$ and $0.874(95 \% \mathrm{Cl}$ 0.822-0.925). Conclusions: The combined use of HBP hypointensity and DWI hyperintensity as a new DC for HCC enables a high diagnostic sensitivity and comparable specificity.

(c) 2020 The Author(s)

Published by S. Karger AG, Basel

\section{Introduction}

Despite the noninvasive diagnosis of hepatocellular carcinoma (HCC), especially in those high-risk patients with typical enhancement pattern, accurate diagnosis of HCC still remains difficult when typical imaging features are lacking $[1,2]$. Unlike Western countries, the incidence of HCC in Asian-Pacific area is mainly based on the chronic hepatitis B virus or hepatitis $C$ virus infection $[3,4]$. In addition, the treatment strategies also vary as the Western countries emphasize more on liver transplantation, and the imaging staging often affects the organ allocation priorities [5, 6]. While hepatectomy, radiofrequency ablation, and transcatheter arterial chemoembolization are more common used in Eastern countries [7, 8]. Therefore, based on the difference of etiology and treatment concept, Western countries more concerned about improving the diagnostic specificity, while Eastern countries pay more attention to the sensitivity of diagnosis [9-11]. Thus, how to effectively improve the specificity of diagnosis while improving the diagnostic sensitivity is an urgent problem.

Typical imaging features including non-rim arterial phase hyperenhancement (APHE) and nonperipheral washout on portal venous phase (PVP) entail high specificity for the diagnosis of HCC and have been recommended as the major imaging features in many Western guidelines like European Association for the Study of the Liver or Liver Imaging Reporting and Data system $[12,13]$. However, typical enhancement pattern will not be presented with some of the early HCC as the abnormal hepatic angiogenesis that has not yet fully formed [14]. In addition, in some benign lesions, such as atypical hemangioma, can also present with typical enhancement pattern of APHE and PVP washout [15]. Gadoxetate acid disodium is a liver specific contrast agent which has been reported to show comparable performance for the assessment of typical enhancement pattern of HCC, in addition, it can also improve the per-lesion detection sensitivity due to its capability for providing tissue-specific hepatobiliary phase (HBP) imaging [16-18]. However, despite HBP showed high sensitivity for lesion detection but nevertheless HBP hypointensity can only serve as an ancillary imaging features in Western guidelines $[12,13]$. One recent study [19] suggested that the use of HBP hypointensity as an alternative to PVP washout and another study [20] showed that HBP hypointensity may be used as an alternative to washout with little loss in specificity after excluding nodules considered to be non-HCC. However, regarding these studies $[19,20]$, the low signal intensity in both PVP and HBP hypointensity related imaging pathology was totally different, that is, the former was based on the abnormal drainage vessels, and the other was based on the function of hepatocyte uptake. Furthermore, in particular, HCC lesions such as some small well-differentiated HCC, it can also present with hyperintensity on HBP [21]. Therefore, relying solely on HBP itself, optimal diagnosis may also be difficult to achieve. Diffusionweighted imaging (DWI) can serve as an important supplement of HBP, as the cell density of HCC is more than twice the density of normal liver parenchymal even in small HCC [22], and HCCs showed hyperintensity on DWI owing to diffusion restriction. To the best of our knowledge, few studies have been performed to prospectively investigate the combination use of ancillary imaging features of Gadoxetic acid-enhanced MRI and DWI as a new diagnostic criterion (DC) for HCC diagnosis.

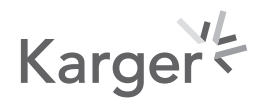


Wei et al.: A New DC with Gadoxetic Acid-Enhanced MRI May Improve the Diagnostic Performance for HCC

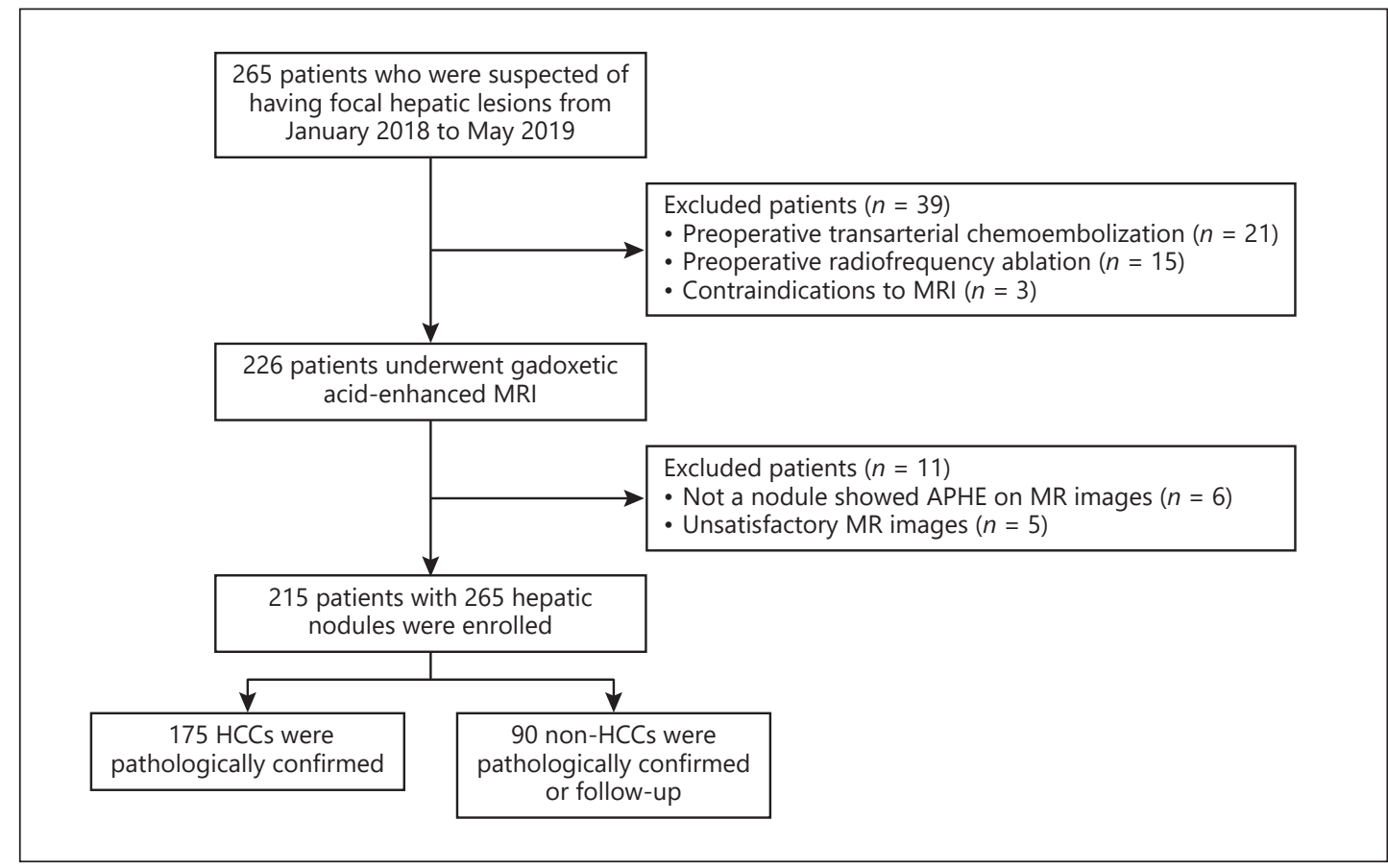

Fig. 1. Flow diagram of patient selection. HCC, hepatocellular carcinoma; APHE, arterial phase hyperenhancement.

Therefore, the purpose of this study was to prospectively evaluate if the combination uses of gadoxetic acid-enhanced MR imaging and DWI as a new DC would improve the diagnostic performance of HCC compared with these typical major imaging features related DC.

\section{Materials and Methods}

\section{Study Population}

This prospective study was approved by the Institutional Review Board, and written informed consent was obtained from all patients. On the basis of expected sensitivity and specificity with an assumed discordant rate of $10 \%$, our power analysis indicated that 170 patients would provide a power of at least $80 \%$ with a one-sided type I error rate of 10\% [23]. Between January 2018 and May 2019, a total of 265 consecutive patients who satisfied the inclusion criteria were potentially enrolled and underwent with Gadoxetic acidenhanced MRI and multi- $b$-value DWI. The inclusion criteria were (1) eligible patients were 18 years or older, (2) patients with the history chronic liver disease (hepatitis B virus or hepatitis C virus infection), (3) focal hepatic lesions identified using ultrasonography or computed tomography, (4) no history of hepatectomy, transcatheter arterial chemoembolization, and radiofrequency ablation, (5) patients showed at least one hepatic nodule with APHE on liver parenchyma, and (6) final diagnosis was confirmed by histopathological analysis or at least 12 months clinical follow-up. Patients were ineligible if they could not provide informed consent, had contraindications to MRI, had poor image quality. Two board-certified abdominal nonblinded reviewers established the reference standard by using marks on all cross-sectional MR images. For participants with multiple hepatic nodules, up to a number of 3 lesions were selected on arterial phase (AP) images by 2 reviewers in a consensus manner. In this study, APHE was defined as nonrim like enhancement in AP unequivocally greater in whole or in part than liver. Restricted diffusion means hyperintensity on DWI and hypointensity on apparent diffusion coefficient map, not attributable solely to T2 shine-restricted diffusion through, unequivocally higher than liver. In addition, lesions on DWI showed target sign (peripheral hyperintensity and central portion hypointensity), HBP targetoid sign (central hyperintensity and peripheral 


\begin{tabular}{|c|c|}
\hline Liver Cancer 2020;9:414-425 & \\
\hline DOI: 10.1159/000505696 & $\begin{array}{l}\text { (c) } 2020 \text { The Author(s). Published by S. Karger AG, Basel } \\
\text { www.karger.com/lic }\end{array}$ \\
\hline
\end{tabular}

\section{Liver Cancer}

Wei et al.: A New DC with Gadoxetic Acid-Enhanced MRI May Improve the Diagnostic Performance for HCC

Table 1. Pulse sequence parameters of gadoxetic acid-enhanced MR images

\begin{tabular}{|c|c|c|c|c|c|c|c|}
\hline Sequence & $\begin{array}{l}\text { Repetition } \\
\text { time, ms }\end{array}$ & $\begin{array}{l}\text { Echo } \\
\text { time, } \\
\text { ms }\end{array}$ & $\begin{array}{l}\text { Field of } \\
\text { view, } \\
\mathrm{cm}^{2}\end{array}$ & $\begin{array}{l}\text { Scan } \\
\text { matrix }\end{array}$ & $\begin{array}{l}\text { Slice } \\
\text { thickness, } \\
\mathrm{mm}\end{array}$ & $\begin{array}{l}\text { Motion } \\
\text { compensation }\end{array}$ & $\begin{array}{l}\text { Fat } \\
\text { saturation }\end{array}$ \\
\hline DISCO & 4.9 & 1.1 & $40 \times 32$ & $320 \times 192$ & 3 & Breath hold & Dixon \\
\hline $\begin{array}{l}\text { LAVA-Flex } \\
\text { In/out of phase }\end{array}$ & 4.5 & 1.8 & $40 \times 32$ & $320 \times 192$ & 4 & Breath hold & Dixon \\
\hline T1-weighted imaging & 150 & $2.4 / 5.8$ & $42 \times 42$ & $288 \times 192$ & 6 & Breath hold & None \\
\hline T2-weighted imaging & $6,500-7,200$ & 83 & $40 \times 40$ & $320 \times 320$ & 6 & $\begin{array}{l}\text { Respiratory } \\
\text { trigger }\end{array}$ & Fat saturation \\
\hline DWI & 7,500 & 77 & $40 \times 30$ & $80 \times 128$ & 6 & $\begin{array}{l}\text { Respiratory } \\
\text { trigger }\end{array}$ & Fat saturation \\
\hline
\end{tabular}

DISCO, differential sub-sampling with cartesian ordering; LAVA, liver acquisition with volume acceleration; DWI, diffusionweighted imaging.

hypointensity), peripheral rim enhancement were classified as non-HCC malignancy. Mild-moderate T2 intensity, mildly or moderately higher than liver hyperintensity and similar to or less than non-iron-overloaded spleen, was also used to exclude these typical marked T2 hyperintensity benign lesions. Detailed information about the inclusion and exclusion criteria was listed in Figure 1.

\section{MR Examination Protocol}

For all participants, MR imaging was carried out by using a 3.0 T MR system (Discovery MR 750w, GE Healthcare, Milwaukee, WI, USA). A 16-channel phased-array torsor coil (GE Medical System) was used for all measurements. All patients were prepared to fast for 6-8 $\mathrm{h}$ before MR examination. In this study, the AP images were achieved by using a multiple AP (MAP) imaging method with differential subsampling cartesian ordering acquisition so as to provide the optimal late AP images and minimize the effect caused by the transient severe motion (TSM) [24]. An 18 s long breath hold with a 20 s delay after the injection of gadoxetate acid disodium (Primovist, Bayer Pharma AG, Berlin, Germany) with the injection rate of $1 \mathrm{~mL} / \mathrm{s}$ was needed. The MAP images were further reconstructed with a temporal resolution of $3 \mathrm{~s}$; thus, 6 consequential phases were obtained at 20,23,26, 29, 32, and 35 s. DWI was acquired with a series $b$ value of $0,200,800$, and 1,000 $\mathrm{mm}^{2} / \mathrm{s}$. For the PVP, delayed phase, and HBP imaging, liver acquisition with volume acceleration-Flex was used for the images acquisition at $60 \mathrm{~s}, 180 \mathrm{~s}$, and $20 \mathrm{~min}$, respectively. Table 1 provides the detailed examination parameters.

\section{Imaging Analysis}

Another 2 board-certified abdominal radiologists independently performed the marked lesion observations while blinded to all clinical data, when the 2 radiologists not agreed with each other, the consensus was reached by discussion. The DCs were subdivided into 5 different diagnostic categories, including DC-1: APHE and PVP washout; DC-2: APHE and HBP hypointensity; DC-3: APHE and DWI hyperintensity; DC-4: HBP hypointensity and DWI hyperintensity; DC-5: HBP hypointensity, DWI hyperintensity and excluded these markedly T2 hyperintensity. According to the different DCs, the 2 radiologists identified these observational nodules as HCC, non-HCC lesions, and the interpretations regarding these imaging features were the same as mentioned above. MR images were presented in a randomized manner, and review of the combined MR images based on different DCs in the same patient was separated by a delay period of 3-4 weeks to minimize recall. In addition, the MR images were distributed by a third radiologist, and there was no time limit for review, the 2 radiologists were also suggested to read the MR images in a clinical manner so that the coronal and sagittal reconstructions can be used.

Determination of Final Diagnosis

Of 265 nodules in 215 patients, 175 nodules were confirmed as HCCs on the basis of pathological evidence (171 nodules were confirmed by using resected specimens, 4 nodules by biopsy). Forty-seven

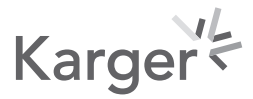




\begin{tabular}{|c|c|}
\hline Liver Cancer 2020;9:414-4 & \\
\hline DOI: 10.1159/000505696 & $\begin{array}{l}\text { (c) } 2020 \text { The Author(s). Published by S. Karger AG, Basel } \\
\text { www.karger.com/lic }\end{array}$ \\
\hline
\end{tabular}

Wei et al.: A New DC with Gadoxetic Acid-Enhanced MRI May Improve the Diagnostic Performance for HCC

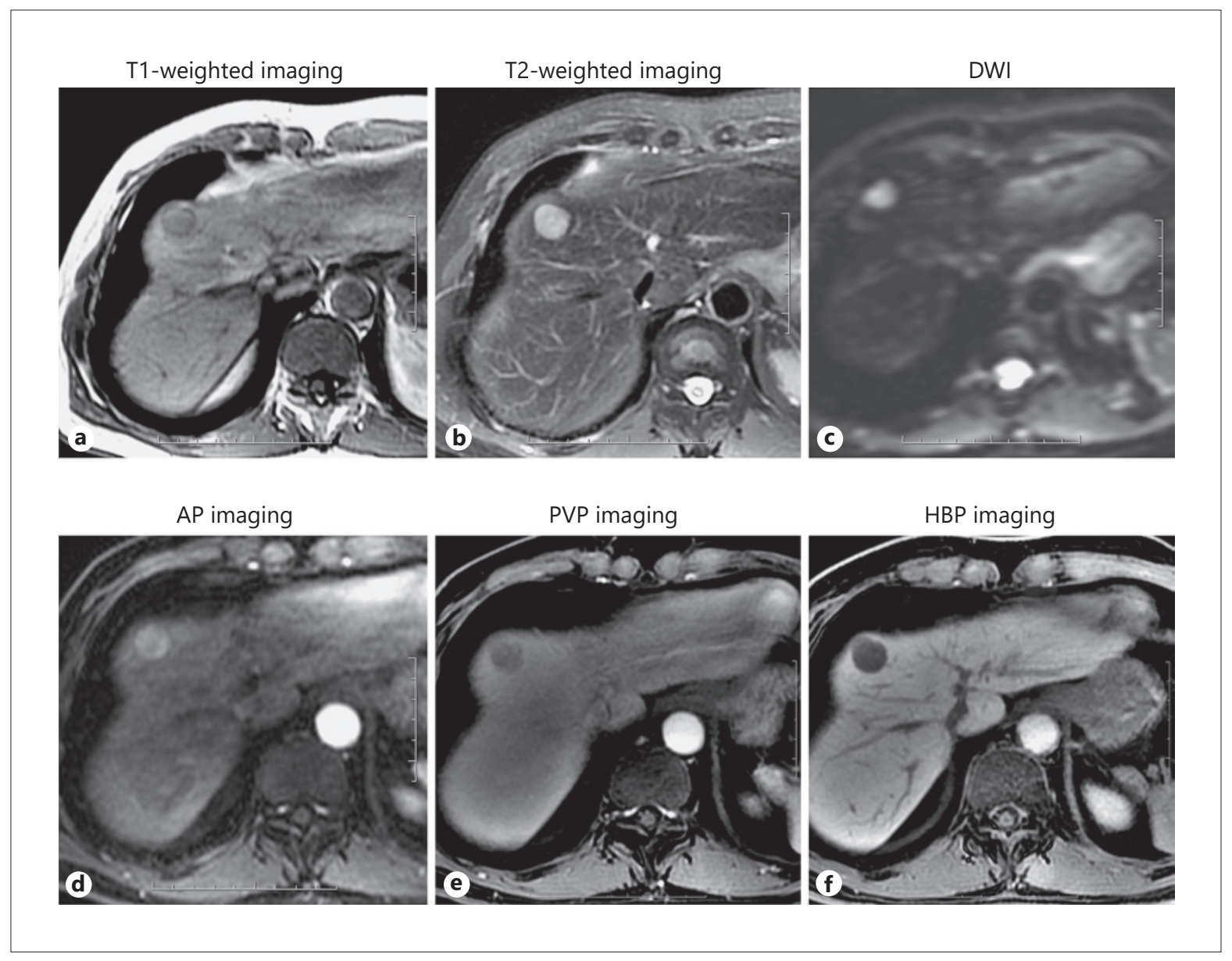

Fig. 2. Surgically confirmed HCC. a T1-weighted imaging; (b) T2-weighted imaging; (c) DWI; (d) AP (imaging); (e) PVP imaging; and (f) HBP imaging. DWI, diffusion-weighted imaging; AP, arterial phase; PVP, portal venous phase; HBP, hepatobiliary phase.

nodules were confirmed as benign lesions by resected specimens (13 high grade dysplastic nodules, 9 regenerative nodules, 5 focal nodular hyperplasias, 6 hepatic adenomas, 2 abscess, 1 hemangioma), biopsy ( 4 HGNDs), or a stable or regressed nodule size for at least 12 months (7 nodules). Additionally, there were 43 other malignant nodules including 2 combined hepatocellular-cholangiocarcinomas, 15 intrahepatic cholangiocarcinomas (ICCs), 8 cholangiocarcinomas, and 18 colorectal liver metastases confirmed by using resected specimens.

\section{Statistical Analysis}

Diagnostic performance of sensitivity, specificity, and accuracy for each imaging diagnostic criteria was calculated. Thereafter, per-lesion diagnostic sensitivity and specificity of imaging criteria were compared by using McNemar's test. $\chi^{2}$ or Fisher exact test was used for the categorical variables. Receiver operating characteristics curves analyses were performed to evaluate the different DCs and the value of area under curve (AUC) was determined. Generalized estimating equations were used to avoid the clustering effect of these multiple lesions in single patient. Kappa statistics were estimated for imaging features evaluation to assess reader agreements, and a kappa value of 0 indicates no agreement, kappa values of 0.01-0.20 represent slight agreement, 0.21-0.40 fair agreement, 0.41-0.60 moderate agreement, 0.61-0.80 good agreement, 0.81-0.99 almost perfect agreement, and 1 perfect agreement. A two-sided $p$ value of $<0.05$ was considered to indicate statistical significance. All statistical analyses were performed by using a statistical software package (SPSS 23.0 [SPSS Inc., Chicago, IL, USA]). 


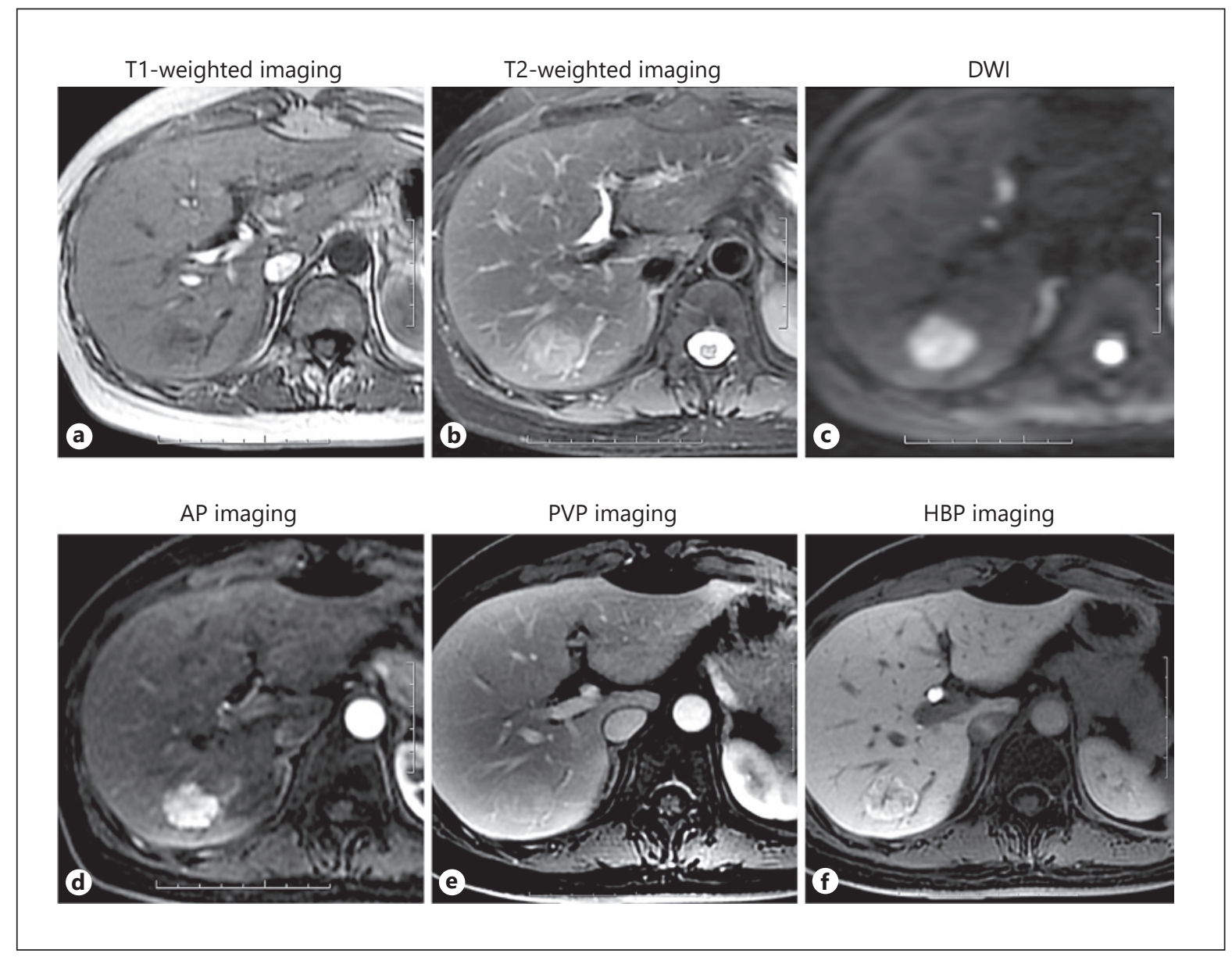

Fig. 3. Surgically confirmed focal nodular hyperplasia. a T1-weighted imaging; (b) T2-weighted imaging; (c) DWI; (d) AP imaging; (e) PVP imaging; and (f) HBP imaging. DWI, diffusion-weighted imaging; AP, arterial phase; PVP, portal venous phase; HBP, hepatobiliary phase.

\section{Results}

\section{Patients Characteristics}

There were 215 patients (mean age $53.82 \pm 11.24$ years; range $24-82$ years) with 265 nodules, including 166 men (54.27 \pm 11.54 years; range $24-82$ years) and 49 women (52.31 \pm 10.14 years; range $32-72$ years). Of these, Hepatitis B was the predominant cause of chronic liver diseases (98.14\%; 211/215), followed by Hepatitis C (1.86\%; 4/215). In addition, 179 patients $(83.26 \% ; 179 / 215)$ had 1 nodule and 36 patients $(16.74 \% ; 36 / 215)$ had 2 or 3 nodules. The 265 nodules consisted of 175 HCCs (Fig. 2) and 90 non-HCC nodules ( 2 abscess, 2 combined hepatocellular-cholangiocarcinomas, 5 focal nodular hyperplasias [Fig. 3], 17 high grade dysplastic nodules, 15 ICCs, 9 regenerative nodules, 8 cholangiocarcinomas, 6 hepatocellular adenomas, 8 hemangiomas, and 18 colorectal liver metastases).

\section{Diagnostic Performance of 5 MR Imaging Features for HCC}

The diagnostic performance of 5 different MR imaging features for HCC diagnosis is summarized in Table 2. Of these 5 MR imaging features, the imaging feature of APHE $(84.00 \%$; 147/175), PVP wash-out (83.43\%; 146/175), HBP hypointensity (97.14\%; 170/175), DWI 
Wei et al.: A New DC with Gadoxetic Acid-Enhanced MRI May Improve the Diagnostic Performance for HCC

Table 2. Performance of 5 different MR imaging features for the diagnosis of HCC

\begin{tabular}{llllllll}
\hline Criterion & $\begin{array}{l}\text { HCC } \\
(n=175)\end{array}$ & $\begin{array}{l}\text { Non-HCC } \\
(n=90)\end{array}$ & $p$ value & $\begin{array}{l}\text { Kappa } \\
\text { value }\end{array}$ & Sensitivity, $\%$ & Specificity, \% & Accuracy, \% \\
\hline APHE & 147 & 38 & $<0.001$ & 0.717 & $\begin{array}{l}84.00(78.51-89.49) \\
{[147 / 175]}\end{array}$ & $\begin{array}{l}57.78(47.38-68.18) \\
{[52 / 90]}\end{array}$ & $\begin{array}{l}75.09(69.85-80.34) \\
{[199 / 265]}\end{array}$ \\
\hline PVP wash-out & 146 & 10 & $<0.001$ & 0.806 & $\begin{array}{l}83.43(77.87-88.99) \\
{[146 / 175]}\end{array}$ & $\begin{array}{l}88.89(82.27-95.51) \\
{[80 / 90]}\end{array}$ & $\begin{array}{l}85.28(80.99-89.58) \\
{[226 / 265]}\end{array}$ \\
\hline $\begin{array}{lllllll}\text { HBP } \\
\text { hypointensity }\end{array}$ & 170 & 32 & $<0.001$ & 0.829 & $\begin{array}{l}97.14(94.65-99.64) \\
{[170 / 175]}\end{array}$ & $\begin{array}{l}64.44(54.36-74.53) \\
{[58 / 90]}\end{array}$ & $86.04(81.84-90.24)$ \\
\hline $\begin{array}{l}\text { DWI } \\
\text { hyperintensity }\end{array}$ & 168 & 41 & $<0.001$ & 0.814 & $\begin{array}{l}96.00(93.07-98.93) \\
{[168 / 175]}\end{array}$ & $\begin{array}{l}54.44(43.96-64.93) \\
{[49 / 90]}\end{array}$ & $\begin{array}{l}81.89(77.22-86.55) \\
{[217 / 265]}\end{array}$ \\
\hline $\begin{array}{l}\text { Mild-moderate } \\
\text { T2 }\end{array}$ & 159 & 48 & $<0.001$ & 0.897 & $\begin{array}{l}90.86(86.54-95.17) \\
{[159 / 175]}\end{array}$ & $\begin{array}{l}46.67(36.16-57.17) \\
{[42 / 90]}\end{array}$ & $75.85(70.66-81.04)$ \\
\hline
\end{tabular}

Numbers in parentheses are $95 \%$ CIs, and numbers in brackets are raw data.

HCC, hepatocellular carcinoma; APHE, arterial phase hyperenhancement; PVP, portal venous phase; HBP, hepatobiliary phase; DWI, diffusion-weighted imaging.

hyperintensity (96.00\%; 168/175), and mild-moderate T2 signal intensity (90.86\%; 159/175) was presented more frequently in HCC group than non-HCC group (All $p<0.001$ ). The interreader agreement for the PVP wash-out, HBP hypointensity, DWI hyperintensity, and mildmoderate $\mathrm{T} 2$ signal intensity was $0.806,0.829,0.814$, and 0.897 , respectively. Good interreader agreement was also obtained for the APHE with the kappa value of 0.717 . For the diagnostic sensitivity, the imaging features of HBP hypointensity and DWI hyperintensity yielded the highest sensitivity of $97.14 \%(170 / 175)$ and $96.00 \%(168 / 175)$, but the imaging feature of PVP wash-out obtained the lowest diagnostic sensitivity of $83.43 \%(146 / 175)$. However, the PVP wash-out was the most specific imaging feature with the specificity of 88.89\% (80/90) and the feature of DWI hyperintensity $(54.44 \% ; 49 / 90)$ and mild-moderate T2 hyperintensity (46.67\%; 42/90) had the lowest diagnostic specificity. Additionally, generalized estimating equation results demonstrated that no clustering effect was obtained with these multiple lesions in single patient (all $|r|<0.001$ ), and 2 imaging features including PVP wash-out (OR 19.028; 95\% CI 7.114-50.890; $p<0.001$ ) and HBP hypointensity (OR 23.843; 95\% CI 6.206-91.610; $p<0.001$ ) were found to be significant in HCC determine after statistical adjustment.

\section{Diagnostic Performance of 5 MRI Criteria for HCC}

The diagnostic performance of $5 \mathrm{MRI}$ criteria for HCC is summarized in Table 3. Of all the 5 different MRI criteria for the diagnosis of HCC, the DC-4 (93.71\%; 164/175) and DC-5 $(92.57 \% ; 162 / 175)$ yielded the highest diagnostic sensitivity of compared with that of DC-1 (72.57\%; 127/175), DC-2 (82.86\%; 145/175), and DC-3 (82.29\%; 144/175; All $p<0.001)$; however, no statistical significance was obtained between DC-4 and DC-5 $(p=0.500)$. For the diagnostic specificity, the DC-1 $(94.44 \% ; 85 / 90)$ achieved the highest diagnostic specificity and subsequent with DC-3 (84.44\%; 76/90), DC-2 (83.33\%; 75/90), DC-5 (82.22\%; 74/90), and DC-4 (74.44\%; 67/90), and statistical significant difference was also obtained between DC-1 versus DC-2 ( $\left.\chi^{2}=5.786, p=0.013\right)$, DC- 1 versus DC-3 $\left(\chi^{2}=4.267, p=0.035\right)$, DC- 1 versus DC-4 $\left(\chi^{2}=13.136, p<0.001\right)$, and DC- 1 versus DC-5 $\left(\chi^{2}=5.882, p=0.013\right)$. In addition, except for the DC-4 versus DC-5 $\left(\chi^{2}=4.000, p=0.039\right)$, no significant differences were obtained for the multiple comparison of the specificity between DC-2, DC 3, DC-4, and DC-5 (all $p>0.05$ ). 


\begin{tabular}{l|l}
\hline Liver Cancer 2020;9:414-425 \\
\hline DOI: 10.1159/000505696 & $\begin{array}{l}\text { @ 2020 The Author(s). Published by S. Karger AG, Basel } \\
\text { www.karger.com/lic }\end{array}$
\end{tabular}

Wei et al.: A New DC with Gadoxetic Acid-Enhanced MRI May Improve the Diagnostic Performance for HCC

Table 3. Performance of 5 different MRI criteria for the diagnosis of HCC

\begin{tabular}{llllll}
\hline Criterion & Sensitivity, $\%$ & Specificity, $\%$ & Accuracy, $\%$ & PPV, $\%$ & NPV, $\%$ \\
\hline DC-1 & $72.57(65.90-79.25)$ & $\begin{array}{l}94.44(89.62-99.27) \\
{[85 / 90]}\end{array}$ & $\begin{array}{l}80.00(75.15-84.85) \\
{[212 / 265]}\end{array}$ & $\begin{array}{l}96.21(92.91-99.51) \\
{[127 / 132]}\end{array}$ & $\begin{array}{l}63.91(55.64-72.18) \\
{[85 / 133]}\end{array}$ \\
\hline DC-2 & $82.86(77.22-88.50)$ & $83.33(75.48-91.18)$ & $83.02(78.47-87.57)$ & $90.63(86.06-95.19)$ & $71.43(62.46-80.21)$ \\
& {$[145 / 175]^{\dagger}$} & {$[75 / 90]^{\ddagger}$} & {$[220 / 265]$} & {$[145 / 160]$} & {$[75 / 105]$} \\
\hline DC-3 & $82.29(76.57-88.00)$ & $84.44(76.81-92.08)$ & $83.02(78.47-87.57)$ & $91.14(86.66-95.62)$ & $71.03(62.29-79.76)$ \\
& {$[144 / 175]^{\dagger}$} & {$[76 / 90]^{\ddagger}$} & {$[220 / 265]$} & {$[144 / 158]$} & {$[76 / 107]$} \\
\hline DC-4 & $93.71(90.08-97.35)$ & $74.44(65.26-83.63)$ & $87.17(83.12-91.22)$ & $87.70(82.95-92.45)$ & $85.90(78.00-93.80)$ \\
& {$[164 / 175]^{\dagger}$} & {$[67 / 90]^{\ddagger}$} & {$[231 / 265]$} & {$[164 / 187]$} & {$[67 / 78]$} \\
\hline DC-5 & $92.57(88.65-96.50)$ & $82.22(74.17-90.27)$ & $89.06(85.27-92.84)$ & $91.01(86.77-95.25)$ & $85.06(77.42-92.70)$ \\
& {$[162 / 175]^{\dagger}$} & {$[74 / 90]^{\ddagger}$} & {$[236 / 265]$} & {$[162 / 178]$} & {$[74 / 87]$} \\
\hline
\end{tabular}

Numbers in parentheses are $95 \%$ CIs, and numbers in brackets are raw data.

${ }^{\dagger}$ Data of the sensitivity and are compared with DC- 1 by using McNemar's test, $p<0.001$.

₹ Data of the specificity are compared with DC-1 by using McNemar's test, $p<0.05$.

HCC, hepatocellular carcinoma; PPV, positive predict value; NPV, negative predict value; DC, diagnostic criterion.

The AUC value for DC-1, DC-2, DC-3, DC-4, and DC-5 was 0.835 (95\% CIs 0.785-0.885), 0.831 (95\% CI 0.776-0.886), 0.834 (95\% CI 0.779-0.888), 0.841 (95\% CI 0.783-0.899), and 0.874 (95\% CI 0.822-0.925), respectively.

\section{Discussion}

In the present study, the difference of 5 imaging features including APHE, PVP wash-out, HBP hypointensity, DWI hyperintensity, and mild-moderate T2 signal intensity was evaluated between HCC and non-HCC group and further the different imaging criterions which contained those imaging features were evaluated. The results demonstrated that the imaging features of HBP hypointensity and DWI hyperintensity were the most sensitive imaging feature for HCC diagnosis, but imaging feature of PVP wash-out yielded the highest diagnostic specificity for HCC. In addition, the results also found that the DC of DC- 4 and DC- 5 yielded the highest AUC value and were the most sensitive DC and the DC- 1 was the most specific criterion for the diagnosis of HCC.

There have been several studies evaluating the diagnostic performance of gadoxetic acid enhanced MR imaging [25-27]. Golfieri et al. [25] found that hypointensity alone in the HBP allowed the identification of the majority of malignant hepatic nodules with atypical enhancement pattern (sensitivity: 88\%; specificity: 97\%), and which is superior to other imaging features in the evaluation of malignant lesions. Choi et al. [26] reported in a retrospective study found that the HBP hypointensity entails a diagnostic sensitivity of $99.5 \%$, and which is also the most sensitive imaging feature among all the evaluated imaging features. The findings of our study also demonstrated that HBP hypointensity seems to be a more sensitive imaging feature for the diagnosis of HCC with the sensitivity of $97.14 \%(170 / 175)$; however, in our study, 5 HCCs $(2.86 \%$; 5/175) with well-differentiated characteristics presented hyperintensity on HBP and which lead to the false negative identification. In addition, it comes to our attention that the imaging feature of DWI hyperintensity entails a high diagnostic sensitivity of $96.00 \%(168 / 175)$ and which is obviously higher than that of the previous studies reported from 75.9 to $87.6 \%[26,28]$. This difference can largely be 
attributed to 2 possible reasons: first, the inclusion criteria bias, as the previous studies mainly included small HCCs $(<3 \mathrm{~cm})$; thus, a high proportion of HCCs especially for those $<1$ $\mathrm{cm}$ would be neglected because of the thickness. Second, the $b$ values selection, the primary goal of this study was to assess the diagnostic sensitivity and specificity. Therefore, a higher $b$ value of $1,000 \mathrm{~mm}^{2} / \mathrm{s}$ was used in this study, not only for improving the sensitivity but also ensuring the specificity. However, in our study, DWI hyperintensity was found not to be specific in HCC diagnosis and which can be explained by the relatively small number of non-HCC nodules and the diffusion restriction on colorectal liver metastasis might further decrease the specificity.

Among the 5 different DCs, our results found that the DC-4 (HBP hypointensity and DWI hyperintensity) and DC-5 (HBP hypointensity, DWI hyperintensity, and excluded these markedly T2 hyperintensity) yielded the highest diagnostic sensitivity compared with other DCs. Joo et al. [19] reported that the DC included hypointensity on HBP as an alternative to PVP washout showed significant higher diagnostic sensitivity (92.8-93.5\%) compared with the DC which only confined to the hypointensity on PVP (70.9\%). Another study reported by Kim et al. [20] also found that the sensitivity with hypointensity extended to HBP (95.2\%) were better than that with hypointensity confined to the PVP (75.7\%), and these results were consistent with our result. According to Liver Imaging Reporting and Data system and European Association for the Study of the Liver guidelines, the AP hyperintensity and PVP washout are suggested as a major DC for HCC diagnosis $[12,13]$. However, the DC may have a debatable premise in common. Our results showed that the DC containing HBP hypointensity and DWI hyperintensity entails high diagnostic sensitivity, and DC containing APHE hypointensity and PVP washout entails low diagnostic sensitivity, and which can be attributed to following 3 explanations: first, unlike the extracellular contrast agent, the hepatocyte uptake of gadoxetic acid for the hepatic specific contrast agent starts with the first pass and is certainly perceivable within the first 90s [29]; thus, the PVP itself on gadoxetic-enhanced MRI should be newly defined and which may low the diagnostic sensitivity of these DC containing APHE and PVP washout. Second, for some early HCCs due to the incomplete neovascularization and for some highly aggressive HCCs due to the conversion of glycolytic metabolism and shut down of the angiogenesis, the APHE may not appear; thus, the imaging feature of APHE has a low sensitivity for HCC diagnosis [30]. Third, due to the high frequency of TSM on AP using hepatic specific contrast agent, the diagnostic sensitivity may be further reduced.

Importantly, excepting for the diagnostic sensitivity, the diagnostic specificity is also of pivotal importance for the hepatic nodules surveillance and treatment decisions [19]. Our results demonstrated that the DC-1 (APHE hyperenhancement and PVP washout) obtained the highest diagnostic specificity of 94.44\% (85/90). Previous studies conducted by Joo et al. [19] and Kim et al. [20] also found that the diagnostic specificity for the same DC was 97.9 and $94.1 \%$, respectively. PVP washout is considered as a strong predictor and major criterion for HCC, in combination with APHE, provides high positive predict value for HCC at risk patients [31]. Interestingly, although the diagnostic specificity of DC-1 is significantly higher than DC-2, DC-3, DC-4, and DC-5 (all $p<0.05$ ), the AUC value of DC-4 and DC-5 was higher than DC-1. The higher AUC value of DC-4 and DC-5 can be explained by the fact that the imaging features these DCs containing. HBP hypointensity is one of the most important imaging features on gadoxetic acid-enhanced MRI and yield high diagnostic sensitivity of HCC; however, some benign lesions such as hemangioma, hepatocellular adenoma would appear hypointensity on HBP. Thus, HBP hypointensity would be more useful for the detection of HCC rather than make the differential diagnosis. By contrast, DWI is a more specific imaging feature for malignant hepatic nodules and using the DWI feature of "target sign" can exclude non-HCC malignant nodules (ICC), therefore, further improving the diagnostic specificity for HCC.

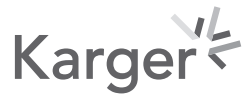


This study has several limitations. First, the different size of the HCCs was not dealt with stratified analysis; thus, it is unclear that whether the size of tumors will influence the diagnostic performance of these imaging criterions. However, previous studies have directly included the hepatic nodules with the size $<3 \mathrm{~cm}$, and the results were identical to our results [26]. Second, due to the high incidence rate of TSM on AP with gadoxetic acid-enhanced MRI, some HCCs might be undetectable on AP and which may further low the diagnostic sensitivity. In our study, MAP imaging was used to improve the detection rate of HCCs; thus, the effect caused by TSM can be reduced to minimum. Finally, the optimal gold standard for this study should be based on a strict imaging-pathology correlation on the explanted liver specimens. However, in our study, not all lesions were pathologically confirmed, resulting in possible over or underestimates of the DCs.

In conclusion, the combined use of HBP hypointensity and DWI hyperintensity as a new DC for HCC enables a high diagnostic sensitivity and comparable specificity when compared with the DC containing APHE and PVP washout. Given the growing use of liver-specific contrast agent, we recommend the HBP hypointensity and DWI hyperintensity as a new DC but not just confined to the traditional DC of APHE and PVP washout.

\section{Acknowledgment}

The authors thank Dongbo Wu, from Department of Infectious disease, West China Hospital, for providing us with some useful suggestions of this manuscript.

\section{Statement of Ethics}

This study was approved by the Institutional Review Board, and written informed consent was obtained from all patients. This study was conducted in accordance with the Declaration of Helsinki.

\section{Disclosure Statement}

None of the authors has conflicts of interest to disclose concerning this study.

\section{Funding Sources}

This work was supported by National Nature Science Foundation of China (Grant number 81771797) and the 1.3.5 project for disciplines of excellence, West China Hospital, Sichuan University (grant number ZYJC18008).

\section{Author Contributions}

All authors participated in the interpretation of study results and in the drafting, critical revision, and approval of the final version of the manuscript. Y.W., Z.Y., Y.Y., H.T., X.H., and B.S. were involved in the study design. Y.W., Z.Y., Z.H., T.Z., and S.W. were investigators in the study and were involved in the data collection. Y.W., Z.Y., and X.W. were involved in the data analysis. B.S. was the medical monitor for the study.

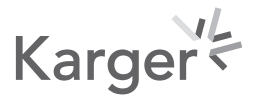


Wei et al.: A New DC with Gadoxetic Acid-Enhanced MRI May Improve the Diagnostic Performance for HCC

\section{References}

1 Heimbach JK, Kulik LM, Finn RS, Sirlin CB, Abecassis MM, Roberts LR, et al. AASLD guidelines for the treatment of hepatocellular carcinoma. Hepatology. 2018 Jan;67(1):358-80.

2 Santillan CS, Tang A, Cruite I, Shah A, Sirlin CB. Understanding LI-RADS: a primer for practical use. Magn Reson Imaging Clin N Am. 2014 Aug;22(3):337-52.

3 de Martel C, Maucort-Boulch D, Plummer M, Franceschi S. World-wide relative contribution of hepatitis B and C viruses in hepatocellular carcinoma. Hepatology. 2015 Oct;62(4):1190-200.

4 Choo SP, Tan WL, Goh BK, Tai WM, Zhu AX. Comparison of hepatocellular carcinoma in Eastern versus Western populations. Cancer. 2016 Nov;122(22):3430-46.

5 Rude MK, Crippin JS. Liver transplantation for hepatocellular carcinoma. Curr Gastroenterol Rep. 2015 Mar; 17(3):11.

6 De Carlis L, Di Sandro S, Centonze L, Lauterio A, Buscemi V, De Carlis R, et al. Liver-allocation policies for patients affected by HCC in Europe. Curr Transplant Rep. 2016 Dec;3(4):313-8.

7 Zhou J, Sun HC, Wang Z, Cong WM, Wang JH, Zeng MS, et al. Guidelines for Diagnosis and Treatment of Primary Liver Cancer in China (2017 Edition). Liver Cancer. 2018 Sep;7(3):235-60.

8 Kudo M, Trevisani F, Abou-Alfa GK, Rimassa L. Hepatocellular Carcinoma: Therapeutic Guidelines and Medical Treatment. Liver Cancer. 2016 Nov;6(1):16-26.

9 Kim TH, Kim SY, Tang A, Lee JM. Comparison of international guidelines for noninvasive diagnosis of hepatocellular carcinoma: 2018 update. Clin Mol Hepatol. 2019 Sep;25(3):245-63.

10 Song P, Cai Y, Tang H, Li C, Huang J. The clinical management of hepatocellular carcinoma worldwide: A concise review and comparison of current guidelines from 2001 to 2017. Biosci Trends. 2017;11(4):389-98.

11 Korean Liver Cancer Association KL, National Cancer Center NC; Korean Liver Cancer Association; National Cancer Center. 2018 Korean Liver Cancer Association-National Cancer Center Korea Practice Guidelines for the Management of Hepatocellular Carcinoma. Gut Liver. 2019 May;13(3):227-99.

12 Galle PR, Forner A, Llovet JM, Mazzaferro V, Piscaglia F, Raoul JL, et al.; European Association for the Study of the Liver. Electronic address: easloffice@easloffice.eu; European Association for the Study of the Liver. Management of hepatocellular carcinoma. J Hepatol. 2018 Jul;69(1):182-236.

13 Kielar AZ, Chernyak V, Bashir MR, Do RK, Fowler KJ, Mitchell DG, et al. LI-RADS 2017: an update. J Magn Reson Imaging. 2018 Jun; 47(6):1459-74.

14 Sano K, Ichikawa T, Motosugi U, Sou H, Muhi AM, Matsuda M, et al. Imaging study of early hepatocellular carcinoma: usefulness of gadoxetic acid-enhanced MR imaging. Radiology. 2011 Dec;261(3):834-44.

15 Klotz T, Montoriol PF, Da Ines D, Petitcolin V, Joubert-Zakeyh J, Garcier JM. Hepatic haemangioma: common and uncommon imaging features. Diagn Interv Imaging. 2013 Sep;94(9):849-59.

16 Haradome H, Unno T, Morisaka H, Toda Y, Kwee TC, Kondo H, et al. Gadoxetic acid disodium-enhanced MR imaging of cholangiolocellular carcinoma of the liver: imaging characteristics and histopathological correlations. Eur Radiol. 2017 Nov;27(11):4461-71.

17 Lee YJ, Lee JM, Lee JS, Lee HY, Park BH, Kim YH, et al. Hepatocellular carcinoma: diagnostic performance of multidetector CT and MR imaging-a systematic review and meta-analysis. Radiology. 2015 Apr;275(1): 97-109.

18 Di Martino M, Marin D, Guerrisi A, Baski M, Galati F, Rossi M, et al. Intraindividual comparison of gadoxetate disodium-enhanced MR imaging and 64-section multidetector CT in the Detection of hepatocellular carcinoma in patients with cirrhosis. Radiology. 2010 Sep;256(3):806-16.

19 Joo I, Lee JM, Lee DH, Jeon JH, Han JK. Retrospective validation of a new diagnostic criterion for hepatocellular carcinoma on gadoxetic acid-enhanced MRI: can hypointensity on the hepatobiliary phase be used as an alternative to washout with the aid of ancillary features? Eur Radiol. 2019 Apr;29(4):1724-32.

20 Kim DH, Choi SH, Kim SY, Kim MJ, Lee SS, Byun JH. Gadoxetic Acid-enhanced MRI of Hepatocellular Carcinoma: Value of Washout in Transitional and Hepatobiliary Phases. Radiology. 2019 Jul;292(1):270.

21 Choi JW, Lee JM, Kim SJ, Yoon JH, Baek JH, Han JK, et al. Hepatocellular carcinoma: imaging patterns on gadoxetic acid-enhanced MR Images and their value as an imaging biomarker. Radiology. 2013 Jun;267(3): 776-86.

22 Nakashima O, Kiyomatsu K, Iemura A, Edamitsu O, Sugihara S, Kojiro M. [Pathomorphologic study on early hepatocellular carcinoma (HCC) — a study of cell density in well-differentiated HCC of the early stage]. Nihon Shokakibyo Gakkai Zasshi. 1990 Jul;87(7):1514-9.

23 Hajian-Tilaki K. Sample size estimation in diagnostic test studies of biomedical informatics. J Biomed Inform. 2014 Apr;48:193-204.

24 Wei Y, Deng L, Yuan Y, Huang Z, He X, Wei X, et al. Gadoxetate acid disodium-enhanced MRI: multiple arterial phases using differential sub-sampling with cartesian ordering (DISCO) may achieve more optimal late arterial phases than the single arterial phase imaging. Magn Reson Imaging. 2019 Sep;61:116-23.

25 Golfieri R, Grazioli L, Orlando E, Dormi A, Lucidi V, Corcioni B, et al. Which is the best MRI marker of malignancy for atypical cirrhotic nodules: hypointensity in hepatobiliary phase alone or combined with other features? Classification after Gd-EOB-DTPA administration. J Magn Reson Imaging. 2012 Sep;36(3):648-57.

26 Choi SH, Byun JH, Lim YS, Yu E, Lee SJ, Kim SY, et al. Diagnostic criteria for hepatocellular carcinoma $\leqslant 3 \mathrm{~cm}$ with hepatocyte-specific contrast-enhanced magnetic resonance imaging. J Hepatol. 2016 May;64(5):1099_ 107. 
27 Usman S, Smith L, Brown N, Major V. Diagnostic accuracy of Magnetic Resonance Imaging using liver tissue specific contrast agents and contrast enhanced Multi Detector Computed Tomography: A systematic review of diagnostic test in Hepatocellular Carcinoma (HCC). Radiography (Lond). 2018 Nov;24(4):e109-14.

28 Kim SS, Kim SH, Song KD, Choi SY, Heo NH. Value of gadoxetic acid-enhanced MRI and diffusion-weighted imaging in the differentiation of hypervascular hyperplastic nodule from small $(<3 \mathrm{~cm})$ hypervascular hepatocellular carcinoma in patients with alcoholic liver cirrhosis: A retrospective case-control study. Journal of magnetic resonance imaging. J Magn Reson Imaging. 2020 Jan;51(1):70-80.

29 Hamm B, Staks T, Mühler A, Bollow M, Taupitz M, Frenzel T, et al. Phase I clinical evaluation of Gd-EOB-DTPA as a hepatobiliary MR contrast agent: safety, pharmacokinetics, and MR imaging. Radiology. 1995 Jun;195(3): 785-92.

30 Tang A, Bashir MR, Corwin MT, Cruite I, Dietrich CF, Do RK, et al.; LI-RADS Evidence Working Group. Evidence Supporting LI-RADS Major Features for CT- and MR Imaging-based Diagnosis of Hepatocellular Carcinoma: A Systematic Review. Radiology. 2018 Jan;286(1):29-48.

31 Zech CJ, Ba-Ssalamah A, Berg T, Chandarana H, Chau GY, Grazioli L, et al. Consensus report from the 8th International Forum for Liver Magnetic Resonance Imaging. Eur Radiol. 2020 Jan;30(1):370-82. 